\title{
Effects of a percutaneous coronary intervention or conservative treatment strategy on treatment outcomes in elderly female patients with acute coronary syndrome
}

DOMAGOJ MARKOVIC ${ }^{1}$, ANDRO RADANOVIC ${ }^{2,}$ ANTE MIHOVILOVIC ${ }^{3}$, JOSKO BOZIC 4 , AJVOR LUKIN², MIROSLAV SIMUNIC

${ }^{1}$ Clinic for heart and cardiovascular diseases, University Hospital of Split, Split, Croatia

${ }^{2}$ Dalmatian County Institute of Emergency Medicine, Croatia

${ }^{3}$ Department of Maxillofacial Surgery, University Hospital of Split, Split, Croatia

${ }^{4}$ Department of Pathophysiology, School of Medicine, University of Split, Split, Croatia

${ }^{5}$ Department of Internal Medicine, University Hospital of Split, Split, Croatia

Corresponding author:

Domagoj Markovic

Clinic for heart and cardiovascular diseases

University Hospital of Split

Spinciceva 1, 21000 Split, Croatia

Phone: $+38591 / 1871771$

E-mail:markovic.domagoj@gmail.com

\section{ABSTRACT}

Aim. To determine the difference in hospital outcomes between percutaneous coronary intervention (PCI) and conservative treatment of elderly female patients hospitalized for acute coronary syndrome (ACS).

Material and Methods. This controlled study included 123 female patients admitted to the Clinic for heart and cardiovascular diseases University Hospital of Split with a diagnosis of ACS and multiple cardiovascular risk factors. We recorded their habits, history, demographics, presenting symptoms, electrocardiograms, ultrasound results, laboratory tests, diagnostic tests and treatment. We compared these data between the two groups, i.e., those treated with conservative therapy and those treated with PCI.

Results. There were fewer arrhythmias $(\mathrm{P}<0.001)$ and episodes of heart failure $(\mathrm{P}<0.001)$ during hospitalization in the PCI group than in the conservative therapy group. There was no significant difference in complications between the groups $(\mathrm{P}=0.887)$.

Conclusion. Elderly female patients with ACS treated with PCI had less arrhythmias and heart failure during hospitalization than those treated with conservative therapy and there was no difference in complications. These results suggest that even high risk patients have better outcomes after treatment with PCI, and therefore PCI is suggested as first-line treatment in these patients, regardless of risk factors.

Key words: percutaneous coronary intervention, acute coronary syndrome, women

\section{INTRODUCTION}

Cardiovascular diseases and especially acute coronary syndrome (ACS) are the leading cause of death in industrialized countries with an incidence of 3 per 1000 inhabitants, and by 2020, are very likely to be so in developing countries as well. (1) Distinguishing patients with ACS among those that present with suspected cardiac pain is a diagnostic challenge, especially in individuals without clear symptoms or electrocardiographic features. Despite modern treatment, mortality and readmission of patients with ACS remains high. (2) With diagnostic advances and treatment of ACS, mortality in men has been reduced over the past decade; however, mortality in women has been increasing since 1984. (3) Previous studies have also shown that women and elderly with ACS are less likely to undergo diagnostic and therapeutic procedures than men. (4-10) Underestimation of risk by the treating physician and atypical presentation could be behind these findings, as well as the presumed increased risks of invasive treatment in women. $(11,12)$ The main reason why physicians choose conservative therapy over percutaneous coronary intervention (PCI) in the elderly is because they are concerned about the complications of an invasive approach in these patients. (13) Recent guidelines from the European Society of Cardiology suggest that age and sex should not be a criterion for conservative therapy because older people and women generally have better outcomes after prompt invasive treatments. (2) Recent studies also show benefits 1 and 5 years after PCI treatment in these patients. $(14,15)$ Limited data, however, exist on in-hospital benefits of invasive therapy, especially in high risk groups. Because elderly female patients with ACS have the least chance of receiving invasive treatment, we wanted to determine whether there is a difference in hospital outcomes between conservative and invasive therapy in these patients.

\section{MATERIAL AND METHODS}

\section{Study population}

This retrospective, historical, controlled study included data from 123 postmeno- 
pausal women with a diagnosis of ACS admitted between February 1st and May 1st, in 2007 or 2011, to the Coronary Care Unit (CCU) of the Clinic for heart and cardiovascular diseases University Hospital of Split. Of the patients admitted during the study period in 2007, 70 were treated with conservative therapy and in 2011, 53 were treated with PCI. Cardiologists made a decision about treatment with the patient's approval. PCI and conservative treatment were administered according to European Society of Cardiology and other relevant guidelines at the time. $(16,17)$

The study was conducted according to the principles of the Declaration of Helsinki. On 16th October, 2010 the Ethics Committee of University Hospital of Split approved the study under the protocol number 2181-147-01/06/J.B.-13-1. The chairperson of the ethics committee was Prof. Jugoslav Bagatin.

\section{Data collection}

Trained research assistants collected demographic data (age, height and weight), socioeconomic status, date of admission, diagnosis on admission, symptoms on admission, physical examination findings on admission, duration of hospitalization, previously diagnosed illnesses, lifestyle habits, marital status, blood test results during hospitalization, clinical examination findings during hospitalization and drugs used before and during hospitalization.

The participants were identified as either suffering from diabetes mellitus or not. Arterial hypertension and year of diagnosis was categorized as previously diagnosed or previous use of antihypertensive drugs. The subjects were identified as either suffering from arterial hypertension or not. In patients with a diagnosis of arterial hypertension we also wrote the year when the arterial hypertension was diagnosed for the first time. Cigarette smoking was categorized as non-smokers or smokers, if they were smoking or had ever smoked. Dyslipidemia was diagnosed as an elevation of total cholesterol $(>4.5 \mathrm{mmol} / \mathrm{L})$, low-density lipoprotein (LDL) cholesterol $(>2.3 \mathrm{mmol} / \mathrm{L})$ and triglyceride (TG) concentrations $(>1.8 \mathrm{mmol} / \mathrm{L})$, and by a de- crease in high-density lipoprotein (HDL) cholesterol concentration in the blood $(<0.9 \mathrm{mmol} / \mathrm{L})$. The subjects were identified as either having dyslipidemia or not. History of coronary artery bypass graft $(\mathrm{CABG})$, ischemic heart disease, cerebrovascular insult, peripheral artery disease, myocardial infarction and PCI were characterized as procedures or diagnoses that the participant had had until the present.

Heart failure was classified using the Killip classification (18) as Killip class 3 or 4 and using the New York Heart Association functional classification (NYHA) (19) as NYHA class 3 or 4. Complications during hospitalization were described as complications that can occur after treatment, modeled by recent guidelines. (2) Heart failure, death and arrhythmias, that can occur as complications of some treatments, were observed separately. Arrhythmia was classified as an abnormal heart rhythm: premature atrial and ventricular contractions, supraventricular tachycardias, ventricular arrhythmias and bradyarrhythmias. Arrhythmia, ST elevation, ST depression, $\mathrm{T}$ waves and $\mathrm{Q}$ waves were diagnosed with the electrocardiograph model ECG-9620M (Nihon Kohden Corporation, Tokyo, Japan). Heart failure, LVEDD and LVEF were diagnosed with a Nemio SSA-550A ultrasound (Toshiba Corporation, Shimoishigami, Japan).

\section{Laboratory Measurements}

Morning blood was collected after 12-hours of fasting and after a 20-minute morning rest. The following parameters were obtained: total cholesterol, LDL, HDL, TG and serum glucose by standard enzymatic methods on an Olympus AU640 (Olympus, Tokyo, Japan). LDL-C was calculated using Friedwald equation. If TG concentration was above $3 \mathrm{mmol} / \mathrm{L}$, HDL-C and LDL-C were measured by direct immunoinhibition method (Olympus Diagnostica, Lismeehan, Ireland) and homogeneous assay (Randox Laboratories, Crumlin, United Kingdom), respectively.

\section{Statistical analysis}

Data analysis was performed using the Statistica 10 software package (StatSoft Inc., Tulsa, OK, USA). Categorical variables were expressed as frequencies and percentages. A chi-square test was used to establish the relationship between pairs of categorical variables. Continuous variables were expressed as mean and standard deviation $(\mathrm{M} \pm \mathrm{SD})$ whereas t-test for independent samples was used to check for statistical significance. The statistical significance level was set at 95\% $(\mathrm{p}<0.05)$.

\section{RESULTS}

One hundred twenty three female patients participated in the study, with a mean age of $71 \pm 8.7$ of those treated conservatively and $65 \pm 8.8$ of those treated with PCI $(\mathrm{P}<0.001$, table 1$)$. There was no significant difference in their body mass index (BMI), systolic blood pressure or diastolic blood pressure, however among those treated with conservative therapy there was significantly less retirees than among those treated with PCI $(\mathrm{n}=9 ; 12.86 \%$ vs $\mathrm{n}=26 ; 52 \%, \mathrm{P}<0.001)$. In the conservative group, the most common symptoms on admission were chest pain $(n=41 ; 58.57 \%$ vs $\mathrm{n}=33 ; 62.26 \%, \mathrm{P}<0.001)$ and dyspnea $(\mathrm{n}=28 ; 40 \%$ vs $\mathrm{n}=7 ; 13.21 \%, \mathrm{P}<0.001)$. In the conservative group there were more patients who smoked ( $n=13 ; 25 \%$ vs $n=7$; $10 \%, \mathrm{P}=0.031)$ and who had dyslipidemia $(\mathrm{n}=33 ; 62 \%$ vs $\mathrm{n}=26 ; 37 \%, \mathrm{P}=0.006$, table 1). Of the laboratory parameters, only triglycerides were significantly higher in participants treated with conservative therapy $(2.21 \pm 1.06)$ compared to those treated with PCI $(1.66 \pm 0.72, \mathrm{P}=0.009$, table 2$)$. There were no statistical differences between heart failure $(\mathrm{n}=31 ; 44.28 \%$ vs $\mathrm{n}=28$; $52.83 \%, \mathrm{P}=0.233$, table 3$)$ and death $(\mathrm{n}=2$; $2.86 \%$ vs $\mathrm{n}=1 ; 1.89 \%, \mathrm{P}=0.73$ ) during admission among the groups, however more arrhythmias were detected in the conservative group ( $n=29,41.43 \%$ vs $n=3,5.66 \%$, $\mathrm{P}<0.001)$ and less ST elevations $(\mathrm{n}=0 ; 0 \%$ vs $\mathrm{n}=9,16.98 \%, \mathrm{P}<0.001)$. Furthermore, during hospitalization there were significantly more cases of heart failure among those treated with conservative therapy $(\mathrm{n}=20 ; 27.4 \%)$ compared to those treated with PCI ( $\mathrm{n}=3$; 5.66\%, $\mathrm{P}=0.001$, table 3$)$. No differences were observed in complications that occurred during hospitalization between the groups. 
Table 1. Characteristics of study patients.

\begin{tabular}{|c|c|c|c|}
\hline & Conservative group $(\mathrm{N}=70)$ & $\mathrm{PCI}$ group $(\mathrm{N}=53)$ & $\mathbf{P}$ \\
\hline Age (years; $M \pm S D$ ) & $71.21 \pm 8.67$ & $65.32 \pm 8.87$ & $<0.001^{\star}$ \\
\hline $\mathrm{BMI}\left(\mathrm{kg} / \mathrm{m}^{2} ; \mathrm{M} \pm \mathrm{SD}\right)$ & $27.5 \pm 8.87$ & $27.51 \pm 4.49$ & 0.989 \\
\hline Systolic BP (mmHg, $\mathrm{M} \pm \mathrm{SD})$ & $154.74 \pm 138.27$ & $141.2 \pm 24.89$ & 0.483 \\
\hline Diastolic BP (mmHg, M \pm SD) & $80.57 \pm 11.25$ & $82.49 \pm 14.17$ & 0.404 \\
\hline Heart rate (beats per minute, $\mathrm{M} \pm \mathrm{SD}$ ) & $80.67 \pm 26.01$ & $72.59 \pm 17$ & 0.055 \\
\hline \multicolumn{4}{|l|}{ Employment: } \\
\hline retirees n (\%) & $9(12.86)$ & $26(52)$ & $<0.001^{*}$ \\
\hline unemployed n (\%) & $39(55.71)$ & $22(41.51)$ & \\
\hline employed n (\%) & $8(11.43)$ & $5(9.43)$ & \\
\hline \multicolumn{4}{|l|}{ Symptoms on admission: } \\
\hline Chest pain $\mathrm{n}(\%)$ & $41(58.57)$ & $33(62.26)$ & $<0.001^{\star}$ \\
\hline Choking n (\%) & $0(0)$ & $3(5.66)$ & \\
\hline Dyspnea n (\%) & $28(40)$ & $7(13.21)$ & \\
\hline Unclear n (\%) & $1(1.42)$ & $10(18.87)$ & \\
\hline Duration of hospitalization (days; $\mathrm{M} \pm \mathrm{SD}$ ) & $6.46 \pm 4.4$ & $5.26 \pm 3.61$ & 0.111 \\
\hline Smoking n (\%) & $7(10)$ & $13(24.53)$ & $0.031^{*}$ \\
\hline Dyslipidemia n (\%) & $26(37.14)$ & $33(62.26)$ & $0.006^{*}$ \\
\hline Diabetes mellitus n (\%) & $14(20)$ & $12(22.64)$ & 0.722 \\
\hline Arterial hypertension n (\%) & $47(67.14)$ & $36(69.92)$ & 0.927 \\
\hline Year of arterial hypertension diagnosis (years; $\mathrm{M} \pm \mathrm{SD}$ ) & $43.45 \pm 18.71$ & $52.5 \pm 8.36$ & 0.087 \\
\hline History of CABG n (\%) & $0(0)$ & $1(1.88)$ & 0.249 \\
\hline History of ischemic heart disease n (\%) & $34(48.57)$ & $26(49.06)$ & 0.957 \\
\hline History of cerebrovascular insult n (\%) & $4(5.71)$ & $3(5.66)$ & 0.99 \\
\hline History of peripheral artery disease n (\%) & $0(0)$ & $1(1.88)$ & 0.249 \\
\hline History of myocardial infarction n (\%) & $13(18.57)$ & $11(20.75)$ & 0.762 \\
\hline History of PCI n (\%) & $6(8.57)$ & $11(20.75)$ & 0.053 \\
\hline
\end{tabular}

$\mathrm{BMI}$, body mass index; $\mathrm{BP}$, blood pressure; $\mathrm{CABG}$, coronary artery bypass graft; $\mathrm{M} \pm \mathrm{SD}$, arithmetic mean \pm standard deviation; $\mathrm{P}$, significance level; ${ }^{*}, \mathrm{P}<0.05$; PCI, percutaneous coronary intervention.

Table 2. Laboratory parameters on admission.

\begin{tabular}{llll}
\hline & Conservative group $(\mathbf{N}=\mathbf{7 0})$ & PCI group $(\mathbf{N}=\mathbf{5 3})$ & P \\
\hline Troponin $(\mu \mathrm{g} / \mathrm{L}, \mathrm{M} \pm \mathrm{SD})$ & $30.29 \pm 72.22$ & $3.2 \pm 7.18$ & 0.074 \\
\hline CKMB $(\mathrm{U} / \mathrm{L}, \mathrm{M} \pm \mathrm{SD})$ & $48.39 \pm 99.46$ & $60.54 \pm 124.38$ & 0.735 \\
\hline $\mathrm{CK}(\mathrm{U} / \mathrm{L}, \mathrm{M} \pm \mathrm{SD})$ & $160.36 \pm 406.17$ & $356.64 \pm 862.48$ & 0.138 \\
\hline Hemoglobin $(\mathrm{g} / \mathrm{L}, \mathrm{M} \pm \mathrm{SD})$ & $129.31 \pm 19.03$ & $128.1 \pm 11.55$ & 0.694 \\
\hline Glucose $(\mathrm{mmol} / \mathrm{L}, \mathrm{M} \pm \mathrm{SD})$ & $7.44 \pm 3.11$ & $7.62 \pm 4.19$ & 0.791 \\
\hline Cholesterol $(\mathrm{mmol} / \mathrm{L}, \mathrm{M} \pm \mathrm{SD})$ & $4.43 \pm 1.24$ & $5.03 \pm 1.37$ & 0.165 \\
\hline Triglycerides $(\mathrm{mmol} / \mathrm{L}, \mathrm{M} \pm \mathrm{SD})$ & $2.21 \pm 1.06$ & $1.66 \pm 0.72$ & $0.009^{*}$ \\
\hline LDL $(\mathrm{mmol} / \mathrm{L}, \mathrm{M} \pm \mathrm{SD})$ & $3.48 \pm 0.88$ & $3.25 \pm 1.06$ & 0.281 \\
\hline HDL $(\mathrm{mmol} / \mathrm{L}, \mathrm{M} \pm \mathrm{SD})$ & $1.4 \pm 0.49$ & $1.33 \pm 0.35$ & 0.498
\end{tabular}

$\overline{\mathrm{CK}}$, creatinine kinase; CKMB, creatinine kinase myoglobin; HDL, high density lipoproteins; LDL, low density lipoproteins; M \pm SD, arithmetic mean \pm standard deviation; $\mathrm{P}$, significance level; ${ }^{*}, \mathrm{P}<0.05$; PCI, percutaneous coronary intervention. 
Table 3. Treatment outcomes.

\begin{tabular}{|c|c|c|c|}
\hline & Conservative group $(\mathrm{N}=70)$ & PCI group $(N=53)$ & $\mathbf{P}$ \\
\hline Heart failure on admission N (\%) & $31(44.28)$ & $28(52.83)$ & 0.233 \\
\hline Death on admission N (\%) & $2(2.86)$ & $1(1.89)$ & 0.73 \\
\hline $\begin{array}{l}\text { Arrhythmias during hospitaliza- } \\
\text { tion N (\%) }\end{array}$ & $29(41.43)$ & $3(5.66)$ & $<0.001^{\star}$ \\
\hline $\begin{array}{l}\text { Heart failure during hospitalization } \\
\mathrm{N}(\%)\end{array}$ & $\mathrm{n} 20(27.4)$ & $3(5.66)$ & $<0.001^{*}$ \\
\hline $\begin{array}{l}\text { Complications during hospitaliza- } \\
\text { tion N (\%) }\end{array}$ & $3(4.29)$ & $2(3.77)$ & 0.887 \\
\hline ST depression on ECG N (\%) & $8(11.43)$ & $5(9.43)$ & 0.722 \\
\hline ST elevation on ECG N (\%) & $0(0)$ & $9(16.98)$ & $<0.001^{\star}$ \\
\hline T wave on ECG N (\%) & $13(18.57)$ & $6(11.32)$ & 0.271 \\
\hline Q wave on ECG N (\%) & $2(2.86)$ & $3(5.66)$ & 0.436 \\
\hline $\operatorname{LVEDD}(\mathrm{mm}, \mathrm{M} \pm \mathrm{SD})$ & $53.48 \pm 5.59$ & $50.41 \pm 7.77$ & 0.275 \\
\hline $\operatorname{LVEF}(\%, \mathrm{M} \pm \mathrm{SD})$ & $48.32 \pm 22.97$ & $59.56 \pm 16.56$ & 0.185 \\
\hline
\end{tabular}

ECG, electrocardiogram; LVEDD, left ventricular end-diastolic dimension; LVEF, left ventricular ejection fraction; $\mathrm{M} \pm \mathrm{SD}$, arithmetic mean \pm standard deviation; $\mathrm{P}$, significance level; ${ }^{\star}, \mathrm{P}<0.05$; PCI, percutaneous coronary intervention.

\section{DISCUSSION}

Women with ACS are more likely to be treated with conservative therapy than men. (4-10) Also, because of possible complication associated with the invasive approach, elderly are less often treated with PCI. (13) Although recent guidelines suggest that women and the elderly have better outcomes after prompt invasive treatments, they are often treated with conservative therapy. (2) Because elderly female patients with ACS have the least chance of getting PCI, we wanted to determine whether there is a difference in hospital outcomes between conservative and PCI in these patients.

In our study, chest pain was present in $59 \%$ of patients on admission. This atypical presentation of patients with ACS has been well documented, especially in the elderly and women. (2) Although patients treated with PCI had more serious presenting symptoms and higher ST elevation during hospitalization, they had better treatment outcomes compared to patients treated with conservative therapy: including less infarctions and less arrhythmias. There was no significant difference in complications during hospitalization after treatment when we compared these two groups, although there were slightly more complications compared to other studies. (20) The reason for this is most likely the higher age of our study groups.

Our study has several limitations. We collected data on patients admitted to only one hospital in 2007 and 2011, and only for several months. The reason we chose 2011 was because that was the first year we had complete data sets of PCI treated patients in those selected months. Another limitation is that we did not analyze the drugs that patients were given during hospitalization, only the type of treatment. Further studies should report on long term effects and include more patients when comparing conservative and PCI in elderly women.
Conclusion of this retrospective study is that elderly female patients presenting with ACS and treated with PCI had significantly less arrhythmias and episodes of heart failure during hospitalization when compared to patients treated with conservative treatment. These results suggest that even high risk patients have better outcomes after treatment with PCI, strengthening PCI as the suggested first-line treatment for ACS, regardless of associated risk factors.

\section{ACKNOWLEDGMENTS}

The authors thank Mario Malicki for his technical support and contribution in data gathering. All authors state that there was no conflict of interest and no funding. All authors have participated in gathering data, processing data, writing the manuscript, approving the last version of the manuscript and submitting the manuscript.

\section{REFERENCES}

1. Murray CJ, Lopez AD. Alternative projections of mortality and disability by cause 1990-2020: Global Burden of Disease Study. Lancet 1997 May 24; 349(9064):1498-504.

2. untitled - guidelines-nste-acs-ft.pdf [Internet]. [Cited 2014 Jun 26]. Available from: http://www.escardio.org/guidelines-surveys/ esc-guidelines/guidelinesdocuments/guidelines-nste-acs-ft.pdf

3. American Heart Association. Heart Disease and Stroke Statistics- 2004 Update. Dallas, TX: American Heart Association, 2003.

4. Ayanian JZ, Epstein AM. Differences in the use of procedures between women and men hospitalized for coronary heart disease. N 
Engl J Med 1991 Jul 25;325(4):221-5.

5. Healy B. The Yentl syndrome. N Engl J Med 1991 Jul 25;325(4):274-6.

6. Poon S, Goodman SG, Yan RT, Bugiardini R, Bierman AS, Eagle KA, et al. Bridging the gender gap: Insights from a contemporary analysis of sex-related differences in the treatment and outcomes of patients with acute coronary syndromes. Am Heart J 2012 Jan;163(1):66-73.

7. Blomkalns AL, Chen AY, Hochman JS, Peterson ED, Trynosky K, Diercks DB, et al, CRUSADE Investigators. Gender disparities in the diagnosis and treatment of non-ST-segment elevation acute coronary syndromes: large-scale observations from the CRUSADE (Can Rapid Risk Stratification of Unstable Angina Patients Suppress Adverse Outcomes With Early Implementation of the American College of Cardiology/American Heart Association Guidelines) National Quality Improvement Initiative. J Am Coll Cardiol 2005 Mar 15;45(6):832-7.

8. Radovanovic D, Erne P, Urban P, Bertel O, Rickli H, Gaspoz J-M. Gender differences in management and outcomes in patients with acute coronary syndromes: results on 20290 patients from the AMIS Plus Registry. Heart 2007 Nov;93(11):1369-75.

9. Ineid H, Fonarow GC, Cannon CP, Hernandez AF, Palacios IF, Maree AO, et al, Get With the Guidelines Steering Committee and Investigators. Sex differences in medical care and early death after acute myocardial infarction. Circulation 2008 Dec 16;118(25):280310.

10. El-Menyar A, Zubaid M, Sulaiman K, AlMahmeed W, Singh R, Alsheikh-Ali AA, Al Suwaidi J. Atypical presentation of acute coronary syndrome: A significant independent predictor of in-hospital mortality. J Cardiol 2011 Mar;57(2):165-71.

11. Xhyheri B, Bugiardini R. Diagnosis and treatment of heart disease: are women different from men? Prog Cardiovasc Dis 2010 Dec;53(3):227-36.

12. Bugiardini R, Bairey Merz CN. Angina with "normal” coronary arteries: a changing philosophy. JAMA J Am Med Assoc 2005 Jan 26;293(4):477-84.

13. Rittger H, Schnupp S, Sinha A-M, Breithardt O-A, Schmidt M, Zimmermann S, et al. Predictors of treatment in acute coronary syndromes in the elderly: impact on decision making and clinical outcome after interventional versus conservative treatment. Catheter Cardiovasc Interv Off J Soc Card Angiogr Interv 2012 Nov 1; 80(5):735-43.

14. Wallentin L, Lagerqvist B, Husted S, Kontny F, Ståhle E, Swahn E. Outcome at 1 year after an invasive compared with a non-invasive strategy in unstable coronary-artery disease: the FRISC II invasive randomised trial. FRISC II Investigators. Fast Revascularisation during Instability in Coronary artery disease. Lancet 2000 Jul 1;356(9223):9-16.

15. Lagerqvist B, Husted S, Kontny F, Ståhle E, Swahn E, Wallentin L. 5-year outcomes in the FRISC-II randomised trial of an invasive versus a non-invasive strategy in non-ST-elevation acute coronary syndrome: a follow-up study. Lancet 2006 Sep 22;368(9540):9981004.

16. Guidelines for the diagnosis and treatment of non-ST-segment elevation acute coronary syndromes - guidelines-NSTE-ACSFT-2007.pdf [Internet]. [Cited 2014 Jun 26]. Available from: http://www.escardio.org/guidelines-surveys/esc-guidelines/Documents/ACS/guidelines-NSTE-ACS-FT-2007.pdf

17. Harlow SD, Gass M, Hall JE, Lobo R, Maki P, Rebar RW, et al, STRAW + 10 Collaborative Group. Executive summary of the Stages of Reproductive Aging Workshop + 10: addressing the unfinished agenda of staging reproductive aging. J Clin Endocrinol Metab 2012 Apr;97(4):1159-68.

18. Killip III T, Kimball JT. Treatment of myocardial infarction in a coronary care unit: A Two year experience with 250 patients. Am J Cardiol 1967 Oct;20(4):457-64.

19. The Criteria Committee of the New York Heart Association Nomenclature and criteria for diagnosis of diseases of the heart and blood vessels. Boston: Little Brown, 1964.

20. Paraschos A, Callwood D, Wightman MB, Tcheng JE, Phillips HR, Stiles GL, et al. Outcomes following elective percutaneous coronary intervention without on-site surgical backup in a community hospital. Am J Cardiol 2005 May 1;95(9):1091-3. 